\title{
Letter
}

\section{Simple cosmological model with inflation and late times acceleration}

\author{
Marek Szydłowski ${ }^{1,2, a}{ }_{\mathbb{D}}$, Aleksander Stachowski ${ }^{1, b}$ \\ ${ }^{1}$ Astronomical Observatory, Jagiellonian University, Orla 171, 30-244 Kraków, Poland \\ ${ }^{2}$ Mark Kac Complex Systems Research Centre, Jagiellonian University, ul. Łojasiewicza 11, 30-348 Kraków, Poland
}

Received: 28 September 2017 / Accepted: 9 March 2018 / Published online: 22 March 2018

(c) The Author(s) 2018

\begin{abstract}
In the framework of polynomial Palatini cosmology, we investigate a simple cosmological homogeneous and isotropic model with matter in the Einstein frame. We show that in this model during cosmic evolution, early inflation appears and the accelerating phase of the expansion for the late times. In this frame we obtain the Friedmann equation with matter and dark energy in the form of a scalar field with a potential whose form is determined in a covariant way by the Ricci scalar of the FRW metric. The energy density of matter and dark energy are also parameterized through the Ricci scalar. Early inflation is obtained only for an infinitesimally small fraction of energy density of matter. Between the matter and dark energy, there exists an interaction because the dark energy is decaying. For the characterization of inflation we calculate the slow roll parameters and the constant roll parameter in terms of the Ricci scalar. We have found a characteristic behavior of the time dependence of density of dark energy on the cosmic time following the logistic-like curve which interpolates two almost constant value phases. From the required numbers of $\mathrm{N}$-folds we have found a bound on the model parameter.
\end{abstract}

\section{Introduction}

While current astronomical observations favour the standard cosmological model [1], the $\Lambda \mathrm{CDM}$ model plays only the role an effective theory of the Universe which offers rather the description of the current properties of the Universe than its explanations. The origin of properties of the current Universe we should find in the very early Universe. In this context a very simple inflation model was proposed by Starobinsky in 1980 [2]. This model attracted attention of cosmologists because it can explain some troubles of the $\Lambda \mathrm{CDM}$ model

\footnotetext{
a e-mail: marek.szydlowski@uj.edu.pl

be-mail: aleksander.stachowski@doctoral.uj.edu.pl
}

in a very simple way. Moreover, this evolutionary scenario is generic and emerged in cosmology in different contexts [1]. In this model, the inflationary scenario of the Universe is driven by the higher quadratic term in the action which takes the form $S=\int \sqrt{-g}\left(R+\frac{R^{2}}{6 M^{2}}\right) \mathrm{d}^{4} x$.

This model $[3,4]$ predicts that the slow roll parameters $n_{s}=1-\frac{2}{N}$ and $r=\frac{12}{N^{2}}$ where $N=50 \sim 60$ is the number of e-folds before the end of inflation, are in good agreement with Planck 2015 data [1].

On the other hand, from the viewpoint of the complete quantum theory of gravity, higher order corrections $\alpha^{\prime}=$ $1 / M_{s}^{2}$ to the Einstein-Hilbert action are always expected i.e.

$$
\begin{aligned}
S= & \int \sqrt{-g}\left(R+c_{2} \alpha^{\prime} R^{2}\right. \\
& +\sum_{i=3} c_{i} \alpha^{\prime i-1} R^{i} \\
& + \text { other higher derivative terms }) \mathrm{d}^{4} x,
\end{aligned}
$$

where $c_{i}$ are the dimensionless couplings.

The higher derivative terms in the action may also originate from supergravity $[5,6]$.

The problem of inflation in polynomial $f(R)$ cosmology was investigated in the metric formalism in [7], where the spectral index and tensor-to-scalar ratio were calculated in the $f(R)$ inflation model.

In this paper we will phenomenologically investigate the inflation model with a polynomial form of the potential in the Palatini formalism in the Einstein frame [8,9]. For simplicity we truncate a Taylor series on the term $R^{3}$.

In the present paper we consider cosmological models of modified gravity which are the polynomial extensions of the Starobinsky model because our aim is to study how tuned is this model and in consequence its prediction-inflation. However, we must remember that the exact form of the function $f(R)$ can be different from such a choice. In particular the adding of negative powers in a $f(R)$ series is also 
very interesting [10]. The treating of the relation $f(R)$ in the form of a series with respect to $R$ guarantees that it is simple enough to handle it easily in the study of physical effects of modified gravity [11]. On the other hand, the introduction of negative powers of $R$ may lead to instabilities [12].

Therefore, it is interesting to investigate some stable isotropic cosmological models describing both inflation and present acceleration in $f(R)$ gravity. In this context the idea of quintessential cosmology seems to be interesting $[10,13]$. In the metric approach a more complicated, non-polynomial form of the function $f(R)$ is required at low curvature [14].

The main aim of the paper is to investigate how rigid the Starobinsky model of inflation is and if it can be disturbed by switching higher order terms. Therefore, our study is motivated by a stability investigation. If the Starobinsky model is stable it is in some sense generic. The standard Starobinsky model of inflation is formulated in the background of a metric formulation of $f(R)$ modified gravity. In this paper we formulate $f(R)$ theory in the Palatini formalism which gives us an equation of motion in the form of a second order equation. The inflation similarly to the Starobinsky approach is obtained after transition to the Einstein frame. We obtain the form of the potential for the scalar field in the covariant form directly parameterized by the Ricci scalar in the Palatini formulation.

We investigate how the shape of the potential changes under changing of the parameter which measures the fraction of the higher order term in the assumed $f(R)$ formula.

In modern cosmology, the Starobinsky model of inflation plays a crucial role [2]. This model of the cosmic inflation is considered as a source of the inflaton field-higher curvature corrections with respect to the Ricci scalar $R$ in the EinsteinHilbert action of gravity of the type $R^{2}$.

The Starobinsky model seems to be distinguished among different alternative models of inflation in predicting a low value of the scalar-to-tensor ratio $r$; namely, it predicts that $r \sim 12 / N^{2}$, where $N$ is the number of e-foldings during inflation [15].

The Starobinsky model is also favoured by experimental results [1,16-19] which give an upper bound on $r$ around the value of 0.1 . What it is important from the observational point of view the Starobinsky model is the model with the highest Bayesian evidence [17]. It is characteristic that the other types of models which also fit the data are actually equivalent to the Starobinsky model during inflation [15].

From the methodological point of view it is important that the Starobinsky model can be embedded in different domains of fundamental physics. The situation is in some sense similar to what happens in mathematics, where an important theorem has many references to it in different areas of mathematics. Here, one can distinguish embedding into the supergravity $[20,21]$ and embedding into the superstring theory [22-26].
In our paper we consider a new embedding of the Starobinsky model into cosmology of Palatini gravity. The emergence of inflation will be demonstrated as an endogenous dynamical effect in the Palatini formulation of gravity applied to FRW cosmology.

\section{Cosmological equations for the polynomial $f(\hat{R})$ theory in the Palatini formalism in the Einstein frame}

In the Palatini formalism, the gravity action for $f(\hat{R})$ gravity has the following form:

$S=S_{\mathrm{g}}+S_{\mathrm{m}}=\frac{1}{2} \int \sqrt{-g} f(\hat{R}) \mathrm{d}^{4} x+S_{\mathrm{m}}$,

where $\hat{R}$ is the generalized Ricci scalar $\hat{R}=g^{\mu \nu} \hat{R}_{\mu \nu}(\hat{\Gamma})$ in the Palatini formalism $[27,28]$. In this approach the torsionless connection $\hat{\Gamma}$ is treated as a variable independent of the spacetime metric $g_{\mu \nu}$ and it is used to construct the Riemann and Ricci tensor.

Let $f^{\prime \prime}(\hat{R}) \neq 0$. In this case, the action (2) has the equivalent form $[11,29,30]$

$$
\begin{aligned}
S\left(g_{\mu \nu}, \Gamma_{\rho \sigma}^{\lambda}, \chi\right)= & \frac{1}{2} \int \mathrm{d}^{4} x \sqrt{-g}\left(f^{\prime}(\chi)(\hat{R}-\chi)\right. \\
& +f(\chi))+S_{m}\left(g_{\mu \nu}, \psi\right) .
\end{aligned}
$$

We introduce a scalar field $\Phi=f^{\prime}(\chi)$, where $\chi=\hat{R}$. Then the action (3) is given by the following form:

$$
\begin{aligned}
S\left(g_{\mu \nu}, \Gamma_{\rho \sigma}^{\lambda}, \Phi\right)= & \frac{1}{2} \int \mathrm{d}^{4} x \sqrt{-g}(\Phi \hat{R}-U(\Phi)) \\
& +S_{m}\left(g_{\mu \nu}, \psi\right),
\end{aligned}
$$

where the potential $U(\Phi)$ is defined as

$U_{f}(\Phi) \equiv U(\Phi)=\chi(\Phi) \Phi-f(\chi(\Phi))$

with $\Phi=\frac{\mathrm{d} f(\chi)}{\mathrm{d} \chi}$ and $\hat{R} \equiv \chi=\frac{\mathrm{d} U(\Phi)}{\mathrm{d} \Phi}$.

The equations of motion are obtained after the Palatini variation of the action (4),

$$
\begin{aligned}
& \Phi\left(\hat{R}_{\mu \nu}-\frac{1}{2} g_{\mu \nu} \hat{R}\right)+\frac{1}{2} g_{\mu \nu} U(\Phi)-T_{\mu \nu}=0, \\
& \hat{\nabla}_{\lambda}\left(\sqrt{-g} \Phi g^{\mu \nu}\right)=0, \\
& \hat{R}-U^{\prime}(\Phi)=0 .
\end{aligned}
$$

From Eq. (6b) we see that a metric connection $\hat{\Gamma}$ is a new (conformally related) metric $\bar{g}_{\mu \nu}=\Phi g_{\mu \nu}$; thus $\hat{R}_{\mu \nu}=$ $\bar{R}_{\mu \nu}, \bar{R}=\bar{g}^{\mu \nu} \bar{R}_{\mu \nu}=\Phi^{-1} \hat{R}$ and $\bar{g}_{\mu \nu} \bar{R}=g_{\mu \nu} \hat{R}$. We can obtain from the $g$-trace of Eq. (6a) a new structural equation,

$$
2 U(\Phi)-U^{\prime}(\Phi) \Phi=T .
$$


Let $\bar{U}(\phi)=U(\phi) / \Phi^{2}, \bar{T}_{\mu \nu}=\Phi^{-1} T_{\mu \nu}$. Then Eq. (6a) and (6c) can be rewritten in the following form:

$$
\begin{aligned}
& \bar{R}_{\mu \nu}-\frac{1}{2} \bar{g}_{\mu \nu} \bar{R}=\bar{T}_{\mu \nu}-\frac{1}{2} \bar{g}_{\mu \nu} \bar{U}(\Phi), \\
& \Phi \bar{R}-\left(\Phi^{2} \bar{U}(\Phi)\right)^{\prime}=0,
\end{aligned}
$$

and we get the following structural equation:

$\Phi \bar{U}^{\prime}(\Phi)+\bar{T}=0$

In this case the action for the metric $\bar{g}_{\mu \nu}$ and the scalar field $\Phi$ has the following form in the Einstein frame:

$$
\begin{aligned}
S\left(\bar{g}_{\mu \nu}, \Phi\right)= & \frac{1}{2} \int \mathrm{d}^{4} x \sqrt{-\bar{g}}(\bar{R}-\bar{U}(\Phi)) \\
& +S_{m}\left(\Phi^{-1} \bar{g}_{\mu \nu}, \psi\right)
\end{aligned}
$$

with a non-minimal coupling between $\Phi$ and $\bar{g}_{\mu \nu}$,

$$
\begin{aligned}
\bar{T}^{\mu \nu} & =-\frac{2}{\sqrt{-\bar{g}}} \frac{\delta}{\delta \bar{g}_{\mu \nu}} S_{m}=(\bar{\rho}+\bar{p}) \bar{u}^{\mu} \bar{u}^{\nu}+\bar{p} \bar{g}^{\mu \nu} \\
& =\Phi^{-3} T^{\mu \nu}
\end{aligned}
$$

$\bar{u}^{\mu}=\Phi^{-\frac{1}{2}} u^{\mu}, \bar{\rho}=\Phi^{-2} \rho, \bar{p}=\Phi^{-2} p, \bar{T}_{\mu \nu}=$ $\Phi^{-1} T_{\mu \nu}, \bar{T}=\Phi^{-2} T$ (see e.g. [30,31]).

We take the metric $\bar{g}_{\mu \nu}$ in the standard form of the FRW metric,

$$
\mathrm{d} \bar{s}^{2}=-\mathrm{d} \bar{t}^{2}+\bar{a}^{2}(\bar{t})\left[\mathrm{d} r^{2}+r^{2}\left(\mathrm{~d} \theta^{2}+\sin ^{2} \theta \mathrm{d} \phi^{2}\right)\right]
$$

where $\mathrm{d} \bar{t}=\Phi(t)^{\frac{1}{2}} \mathrm{~d} t$ and a new scale factor $\bar{a}(\bar{t})=$ $\Phi(\bar{t})^{\frac{1}{2}} a(\bar{t})$. We assume the cosmological equations for the barotropic matter in the following form:

$$
\begin{aligned}
3 \bar{H}^{2} & =3\left(\frac{\dot{\bar{a}}}{\overline{\bar{a}}}\right)^{2}=\bar{\rho}_{\Phi}+\bar{\rho}_{\mathrm{m}}+\Lambda, \quad 6 \frac{\ddot{\bar{a}}}{\overline{\bar{a}}} \\
& =2 \bar{\rho}_{\Phi}-\bar{\rho}_{\mathrm{m}}(1+3 w)
\end{aligned}
$$

where

$\bar{\rho}_{\Phi}=\frac{1}{2} \bar{U}(\Phi), \quad \bar{\rho}_{\mathrm{m}}=\rho_{0} \bar{a}^{-3(1+w)} \Phi^{\frac{1}{2}(3 w-1)}$

and $w=\bar{p}_{\mathrm{m}} / \bar{\rho}_{\mathrm{m}}=p_{\mathrm{m}} / \rho_{\mathrm{m}}$. The conservation equation has the following form:

$\dot{\bar{\rho}}_{\mathrm{m}}+3 \bar{H} \bar{\rho}_{\mathrm{m}}(1+w)=-\dot{\bar{\rho}}_{\Phi}$

In this paper, we consider the Palatini model, $f(\hat{R})=$ $\sum_{i=1}^{n} \gamma_{i} \hat{R}^{i}$, in the Einstein frame, where $\gamma_{1}=1$. In this case, the potential $\bar{U}$ is given by the following formula:

$\bar{U}(\hat{R})=2 \bar{\rho}_{\Phi}(\hat{R})=\frac{\sum_{i=1}^{n}(i-1) \gamma_{i} \hat{R}^{i}}{\left(\sum_{i=1}^{n} i \gamma_{i} \hat{R}^{i-1}\right)^{2}}$ and the scalar field $\Phi$ has the following form:

$\Phi(\hat{R})=\frac{\mathrm{d} f(\hat{R})}{\mathrm{d} \hat{R}}=\sum_{i=1}^{n} i \gamma_{i} \hat{R}^{i-1}$

\section{Inflation in $f(\hat{R})=\hat{R}+\gamma \hat{R}^{2}+\delta \hat{R}^{3}$ theory in the Palatini formalism in the Einstein frame}

Let $f(\hat{R})=\hat{R}+\gamma \hat{R}^{2}+\delta \hat{R}^{3}$. For this case

$\bar{U}(\hat{R})=\frac{\hat{R}^{2}(\gamma+2 \delta \hat{R})}{\left(1+2 \gamma \hat{R}+3 \delta \hat{R}^{2}\right)^{2}}$

and

$\Phi=1+2 \gamma \hat{R}+3 \delta \hat{R}^{2}$

For this parameterization, we can obtain, from the structural equation (10), a parameterization of $\bar{\rho}_{\mathrm{m}}$ with respect to $\hat{R}$,

$\bar{\rho}_{\mathrm{m}}(\hat{R})=\frac{\hat{R}-\delta \hat{R}^{3}}{\left(1+2 \gamma \hat{R}+3 \delta \hat{R}^{2}\right)^{2}}-4 \Lambda$.

In consequence, the Friedmann equation is given by the following equation:

$$
\begin{aligned}
3 \bar{H}^{2} & =\bar{\rho}_{\mathrm{m}}(\hat{R})+\frac{\bar{U}(\hat{R})}{2}+\Lambda \\
& =\frac{\hat{R}(2+\gamma \hat{R})}{2\left(1+2 \gamma \hat{R}+3 \delta \hat{R}^{2}\right)^{2}}-3 \Lambda .
\end{aligned}
$$

As a reminder, the Hubble function in the Einstein frame $\bar{H}$ is defined by Eq. (14) and the generalized Ricci scalar in the Palatini formalism is $\hat{R}=g^{\mu \nu} \hat{R}_{\mu \nu}(\hat{\Gamma})$.

In this model inflation appears when matter $\bar{\rho}_{\mathrm{m}}$ is negligible with comparison to $\bar{\rho}_{\phi}$.

In the statistical analysis the slow roll parameters are helpful in the estimation of the model parameter in the inflation period [1]. These parameters are defined as

$\epsilon=-\frac{\dot{H}}{H^{2}} \quad$ and $\quad \eta=2 \epsilon-\frac{\dot{\epsilon}}{2 H \epsilon}$.

In our model the slow roll parameters have the following form in the case when $\delta=0$ :

$\epsilon=\frac{3}{2} \frac{\hat{R}-4 \Lambda(1+2 \gamma \hat{R})^{2}}{\hat{R}+\frac{\gamma}{2} \hat{R}^{2}-3 \Lambda(1+2 \gamma \hat{R})^{2}}$,

$\eta=5+\frac{3}{2(\gamma \hat{R}-1)}+\frac{\hat{R}(1+2 \gamma \hat{R})}{6 \Lambda(1+2 \gamma \hat{R})^{2}-\hat{R}(2+\gamma \hat{R})}$. 
From the Planck observations, we know the limits at a 2$\sigma$ level of the values of the scalar spectral index $n_{\mathrm{s}}$ and the tensor-to-scalar ratio $r\left(n_{\mathrm{s}}=0.9667 \pm 0.0040\right.$ and $r<0.113$ [1]). The relations between the scalar spectral index and the tensor-to-scalar ratio and the slow roll parameters are the following:

$n_{\mathrm{s}}-1=-6 \epsilon+2 \eta$ and $r=16 \epsilon$.

Because the slow roll parameters $\epsilon$ and $\eta$ cannot be treated as constant parameters in our model (see Figs. 1 and 2), we cannot use these parameters to find the restriction on the parameter $\gamma$ from astronomical observations [1].

For example, if we assume that $\frac{\Lambda}{3 H_{0}^{2}}=0.6911$, where $H_{0}=67.74 \frac{\mathrm{km}}{\mathrm{s} \mathrm{Mpc}}[1]$, then we get $3.277 \times 10^{-6} \frac{\mathrm{s}^{2} \mathrm{Mpc}^{2}}{\mathrm{~km}^{2}}<$ $\gamma<3.285 \times 10^{-6} \frac{\mathrm{s}^{2} \mathrm{Mpc}^{2}}{\mathrm{~km}^{2}}, 0<\Omega_{\mathrm{m}}=\frac{\bar{\rho}_{\mathrm{m}}}{3 \bar{H}^{2}}<0.0047$ and $\Omega_{\Phi}=\frac{\bar{\rho}_{\Phi}}{3 \bar{H}^{2}} \approx 0.50$. But this value of the parameter $\gamma$ is too large for explaining the present evolution of the Universe. In consequence, the slow roll parameters are useless in the estimation of the parameter $\gamma$.

The slow roll parameter approximation is more restrictive than the constant roll condition $[32,33]$. The constant roll condition has the following form:

$\beta=\frac{\ddot{\Phi}}{\bar{H} \dot{\Phi}}=$ const.

When $\beta \ll 1$ then we get the slow roll approximation.

In our case $\frac{\ddot{\Phi}}{\bar{H} \dot{\Phi}}$ is given by

$$
\begin{aligned}
\frac{\ddot{\Phi}}{\bar{H} \dot{\Phi}}= & -240 \gamma \Lambda+\frac{2}{1-24 \gamma \Lambda}-192 \gamma^{2} \Lambda \hat{R} \\
& +\frac{9(36 \gamma \Lambda-1)}{(\gamma \hat{R}-1)^{2}} \\
& +\frac{12 \Lambda+3(8 \gamma \Lambda-1) \hat{R}}{(24 \gamma \Lambda-1)(6 \Lambda+\hat{R}(24 \gamma \Lambda-2+\gamma(24 \gamma \Lambda-1) \hat{R}))},
\end{aligned}
$$

when $\delta=0 . \frac{\ddot{\Phi}}{\bar{H} \dot{\Phi}}$ is not constant (see Fig. 3) at all times, but beyond the logistic-like type transition it can be well approximated by a constant value. At this intermediate interval the effects of matter do not become negligible. The constant roll inflation approximation is approximately valid beyond a short time during which the effects of matter stay very important (in consequence of the interaction between matter and dark energy).

Figure 1 presents the evolution of $\epsilon$ with respect to the cosmological time $\bar{t}$. We can see that $\epsilon$ is not a constant function when matter is not negligible (see Fig. 4).

Figure 2 demonstrates the evolution of $\eta$ with respect to the cosmological time $\bar{t}$. Note that $\eta$ is not a constant function when matter is not negligible (see Fig. 4). The characteristic

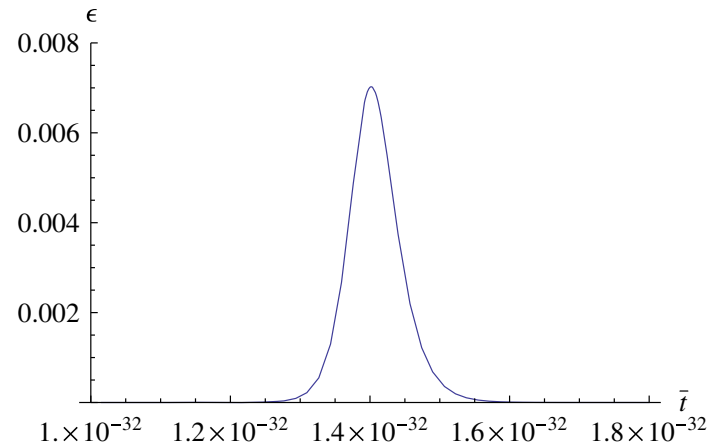

Fig. 1 The diagram presents the evolution of $\epsilon$ with respect to the cosmological time $\bar{t}$. The time is expressed in seconds. The value of the parameter $\gamma$ is assumed as $3.277 \times 10^{-6} \frac{\mathrm{s}^{2} \mathrm{Mpc}^{2}}{\mathrm{~km}^{2}}$. We also assume that $\frac{\Lambda}{3 H_{0}^{2}}=0.6911$, where $H_{0}=67.74 \frac{\mathrm{km}}{\mathrm{s} \mathrm{Mpc}}$. Note that $\epsilon$ is not a constant function when matter is not negligible (see Fig. 4)

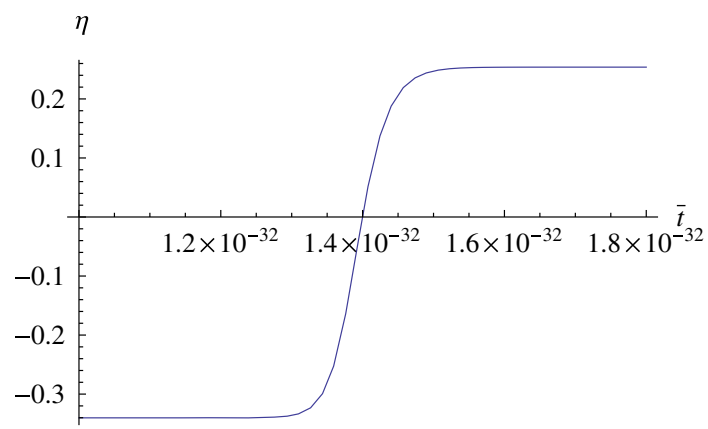

Fig. 2 The evolution of $\eta$ with respect to the cosmological time $\bar{t}$. The time is expressed in seconds. The value of the parameter $\gamma$ is assumed as $3.277 \times 10^{-6} \frac{\mathrm{s}^{2} \mathrm{Mpc}^{2}}{\mathrm{~km}^{2}}$. We also assume that $\frac{\Lambda}{3 H_{0}^{2}}=0.6911$, where $H_{0}=67.74 \frac{\mathrm{km}}{\mathrm{s} \mathrm{Mpc}}$. Note that $\eta$ is not a constant function when matter is not negligible (see Fig. 4). It is interesting that the function $\eta$ is of logistic-like function type

attribute of the function $\eta$ is the shape of the logistic-like function.

Figure 3 presents the evolution of $\frac{\ddot{\Phi}}{\bar{H} \dot{\Phi}}$ with respect to the cosmological time $\bar{t}$. It is important that $\frac{\ddot{\Phi}}{\bar{H} \dot{\Phi}}$ is not a constant function when matter is not negligible (see Fig. 4). It is interesting that the $\frac{\ddot{\Phi}}{\bar{H} \dot{\Phi}}$ function is of the logistic-like function type.

Note that $\beta=\frac{\mathrm{d} \ln \dot{\Phi}}{\mathrm{d} \ln a}=\frac{\ddot{\Phi}}{\bar{H} \dot{\Phi}}$ measures the elasticity of $\dot{\Phi}$ with respect to the scale factor. When $\beta$ is constant then

$\dot{\Phi} \propto a^{\beta}$.

Therefore, if $\beta$ is positive then $\dot{\Phi}$ is a growing function of the scale factor. In the opposite case $(\beta<0) \dot{\Phi}$ is an increasing function of the scale factor and goes to zero for large values of the scale factor. 


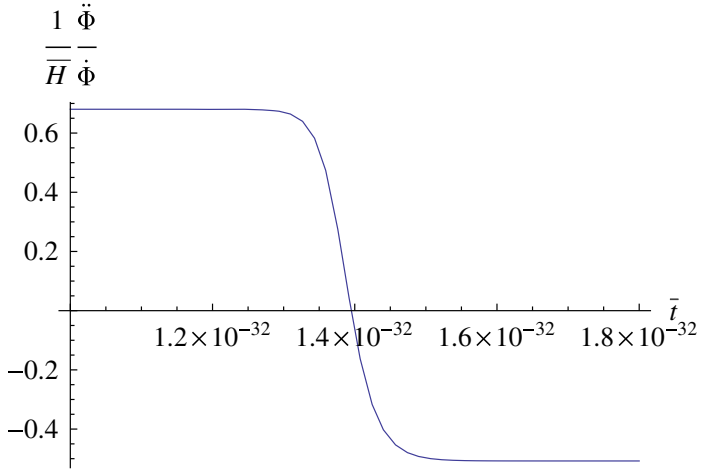

Fig. 3 The diagram presents the evolution of $\frac{\ddot{\phi}}{\bar{H} \dot{\Phi}}$ with respect to the cosmological time $\bar{t}$. The time is expressed in seconds. The value of the parameter $\gamma$ is assumed as $3.277 \times 10^{-6} \frac{\mathrm{s}^{2} \mathrm{Mpc}^{2}}{\mathrm{~km}^{2}}$. We also assume that $\frac{\Lambda}{3 H_{0}^{2}}=0.6911$, where $H_{0}=67.74 \frac{\mathrm{km}}{\mathrm{s} \mathrm{Mpc}}$. Note that $\frac{\ddot{\phi}}{\bar{H} \dot{\Phi}}$ is not a constant function when matter is not negligible (see Fig. 4). It is interesting that $\frac{\ddot{\phi}}{\tilde{H} \dot{\Phi}}$ function is of the logistic-like function type

The slow roll approximation is achieved in our model when matter is negligible. Of course, the constant roll condition is respected automatically.

The evolution of matter in the inflation period can be divided into four phases. The first phase is when matter is negligible and the density of $\bar{\rho}_{\mathrm{m}}$ increases by the interaction with the potential $\bar{\rho}_{\Phi}$. The second phase is when the matter cannot be negligible and its density still increases. In this phase the injection of matter is the most effective. After achieving of the maximum of the density of $\bar{\rho}_{\mathrm{m}}$ the third phase appears. In this phase matter still cannot be negligible but its density decreases. The last phase is when matter density decreases and is negligible.

The evolution of matter in the inflation period is presented in Fig. 4. We see all four phases of the evolution of matter. The maximum is achieved when

$\hat{R}=\frac{1}{2 \gamma}$.

In the maximum, the value of $\bar{\rho}_{\mathrm{m}}$ is equal to $\frac{1}{8 \gamma}-4 \Lambda$.

In detail, the behaviour of the potential function $\bar{U}(\Phi)$ depends on the form of $f(\hat{R})$. For the polynomial form of $f(\hat{R})$, there are two cases. In the first case $f(\hat{R})$ is in the form $f(\hat{R})=\hat{R}+\gamma \hat{R}^{2}$. The typical behaviour of the potential $\bar{U}(\Phi)$ for $f(\hat{R})=\hat{R}+\gamma \hat{R}^{2}$ is present in Fig. 5. The characteristic attribute is a plateau for a large value of $\Phi$ like for the Starobinsky potential [2]. In this case the formula for the potential $\bar{U}(\Phi)$ has the following form:

$\bar{U}(\Phi)=\gamma\left(\frac{\Phi-1}{2 \gamma \Phi}\right)^{2}$

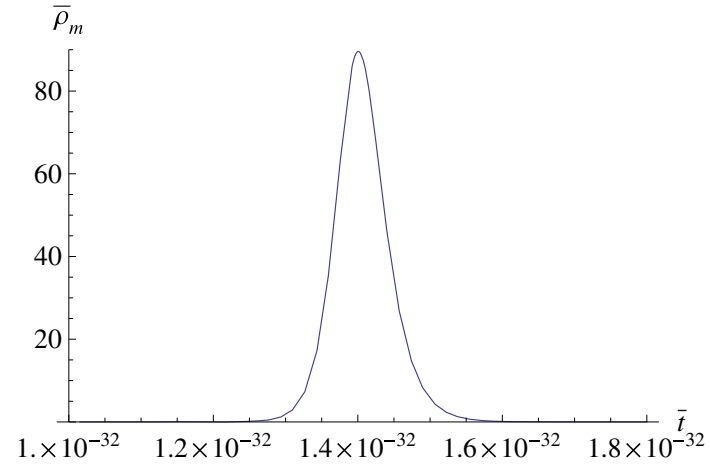

Fig. 4 The diagram presents the evolution of $\bar{\rho}_{\mathrm{m}}$ with respect to the cosmological time $\bar{t}$. The time is expressed in seconds and $\bar{\rho}_{\mathrm{m}}$ is expressed in $\frac{\mathrm{km}^{2}}{\mathrm{~s}^{2} \mathrm{Mpc}^{2}}$. The value of the parameter $\gamma$ is assumed as $3.277 \times 10^{-6} \frac{\mathrm{s}^{2} \mathrm{Mpc}^{2}}{\mathrm{~km}^{2}}$. We also assume that $\frac{\Lambda}{3 H_{0}^{2}}=0.6911$, where $H_{0}=67.74 \frac{\mathrm{km}}{\mathrm{s} \mathrm{Mpc}}$. Note that the maximum of this function is achieved when $\hat{R}=\frac{1}{2 \gamma}$

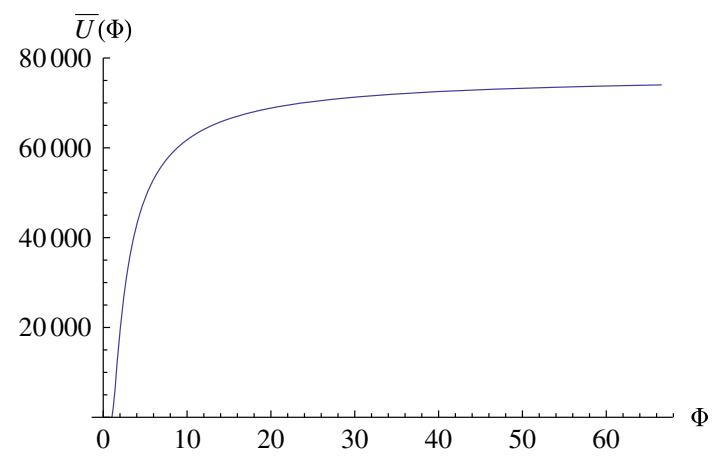

Fig. 5 The diagram presents the typical behaviour of the function $\bar{U}(\Phi)$ for the case $f(\hat{R})=\hat{R}+\gamma \hat{R}^{2}$. The potential $\bar{U}(\Phi)$ is expressed in $\frac{\mathrm{km}^{2}}{\mathrm{~s}^{2} \mathrm{Mpc}^{2}}$. Note that, for the large value of $\Phi$, the function $\bar{U}(\Phi)$ has the plateau

The second case is when $f(\hat{R})$ is of the form $f(\hat{R})=$ $\hat{R}+\gamma \hat{R}^{2}+\sum_{i=2}^{n} \delta_{i} \hat{R}^{i+1}$. Then the potential $\bar{U}(\Phi)$ has no plateau and decreases asymptotically to zero when $\Phi$ goes to infinity. This situation is presented in Fig. 6. The formula for the potential $\bar{U}(\Phi)$ for $f(\hat{R})=\hat{R}+\gamma \hat{R}+\delta \hat{R}^{2}$ has the following form:

$$
\begin{aligned}
& \bar{U}(\Phi) \\
& =\frac{\left(\gamma-\sqrt{\gamma^{2}+3 \delta(\Phi-1)}\right)^{2}\left(\gamma+2 \sqrt{\gamma^{2}+3 \delta(\Phi-1)}\right)}{27 \delta^{2} \Phi^{2}} .
\end{aligned}
$$

In the context of inflation Ijjas et al. [34] pointed out the problem with the desired plateau in the behaviour of the potential of the scalar field. Such a choice seems to be unjustified because it requires that the power series expansion of potential $U$ with respect to $\Phi$ is cancelled at a precise order in $\Phi$ to make the plateau appear. 


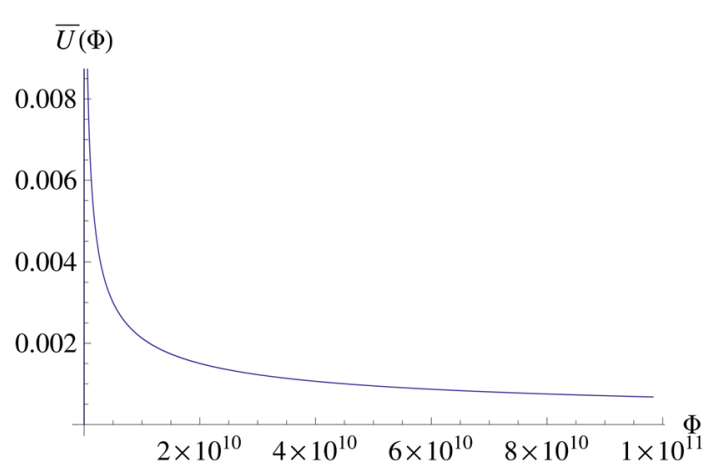

Fig. 6 The diagram presents the typical behaviour of the function $\bar{U}(\Phi)$ for the case $f(\hat{R})=\hat{R}+\gamma \hat{R}^{2}+\delta \hat{R}^{2}$. The potential $\bar{U}(\Phi)$ is expressed in $\frac{\mathrm{km}^{2}}{\mathrm{~s}^{2} \mathrm{Mpc}^{2}}$. Note that, for a large value of $\Phi$, the function $\bar{U}(\Phi)$ decreases asymptotically to zero

In agreement with Ijjas et al. we obtain the plateau of the potential $\bar{U}(\Phi)$ only when $f(\hat{R})=\hat{R}+\gamma \hat{R}^{2}$. For the higher order terms in the expansion of the $f(\hat{R})$, i.e., $\hat{R}^{3}$ and higher, the potential monotically decreases to zero.

Now, we consider in detail inflation in the two abovementioned cases with the potential expanded to second order and third order with respect to $\Phi$. In consequence, we study whether the plateau is necessary for the appearance of inflation in our model and whether inflation is possible for the model with a cut-off in a higher order $\left(\hat{R}^{3}\right.$ and higher) expansion.

In the inflation period when the matter is negligible, the Ricci scalar $\hat{R}$ is constant. The evolution of the Ricci scalar $\hat{R}$ is presented in Fig. 7. We can see three phases of the evolution of the Ricci scalar $\hat{R}$. The first phase is when matter is negligible and the density of $\bar{\rho}_{\mathrm{m}}$ is increased by an interaction with the potential $\bar{\rho}_{\Phi}$. Then the Ricci scalar $\hat{R}$ is constant and is described by the following formula when $\delta=0$ :

$\hat{R}=\frac{1-16 \gamma \Lambda+\sqrt{1-32 \gamma \Lambda}}{32 \gamma^{2} \Lambda}$.

The second phase is when the matter is not negligible. In this case, the Ricci scalar $\hat{R}$ decreases. The last phase is when matter density decreases and is negligible. Then the Ricci scalar $\hat{R}$ is constant and is equal to

$\hat{R}=\frac{1-16 \gamma \Lambda-\sqrt{1-32 \gamma \Lambda}}{32 \gamma^{2} \Lambda}$,

when $\delta=0$. The function which describes the evolution of the Ricci scalar $\hat{R}$ has the shape of a logistic-like function.

The evolution of $\bar{\rho}_{\Phi}$, in the inflation period, similar qualitatively to the evolution of the Ricci scalar $\hat{R}$. We can find three phases. In the first phase, $\bar{\rho}_{\Phi}$ is constant and is equal to

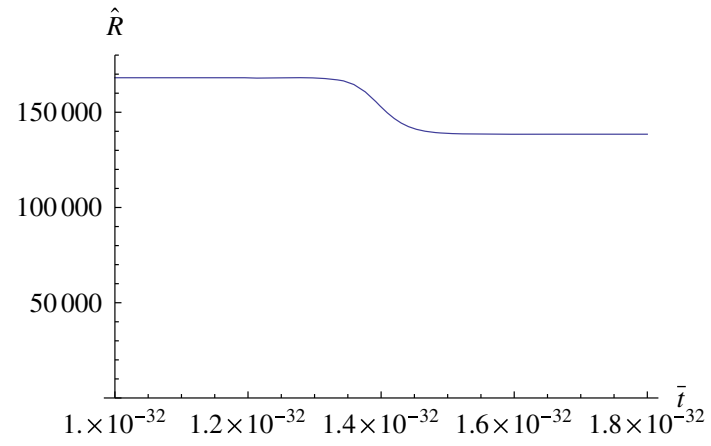

Fig. 7 The diagram presents the evolution of the Ricci scalar $\hat{R}$ with respect to the cosmological time $\bar{t}$. The time is expressed in seconds and the Ricci scalar $\hat{R}$ is expressed in $\frac{\mathrm{km}^{2}}{\mathrm{~s}^{2} \mathrm{Mpc}^{2}}$. The value of the parameter $\gamma$ is assumed as $3.277 \times 10^{-6} \frac{\mathrm{s}^{2} \mathrm{Mpc}^{2}}{\mathrm{~km}^{2}}$. We also assume that $\frac{\Lambda}{3 H_{0}^{2}}=$ 0.6911 , where $H_{0}=67.74 \frac{\mathrm{km}}{\mathrm{s} \mathrm{Mpc}}$. The transition phase is of logistic-like behaviour and is strictly correlated with a peak of the matter density, as shown in Fig. 4

$\bar{\rho}_{\Phi}=\frac{1-16 \gamma \Lambda+\sqrt{1-32 \gamma \Lambda}}{16 \gamma}$

and in the last phase when $\bar{\rho}_{\Phi}$ is also constant,

$\bar{\rho}_{\Phi}=\frac{1-16 \gamma \Lambda-\sqrt{1-32 \gamma \Lambda}}{16 \gamma}$

for $\delta=0$. The difference between $\bar{\rho}_{\Phi}$ in the first and in the last phase is equal to

$\Delta \bar{\rho}_{\Phi}=\frac{\sqrt{1-32 \gamma \Lambda}}{8 \gamma} \approx \frac{1}{8 \gamma}$.

The evolution of $\bar{\rho}_{\Phi}$ is presented in Fig. 8. Our model predicts a phase of the early constant dark energy which is correlated with inflation $[35,36]$.

When $\delta=0$ the number of e-folds in the first phase is equal to

$N=\frac{1}{4 \sqrt{3}} \sqrt{\frac{1+\sqrt{1-32 \gamma \Lambda}}{\gamma}}\left(\bar{t}_{\text {fin }}-\bar{t}_{\text {ini }}\right) \approx \frac{\bar{t}_{\text {fin }}-\bar{t}_{\text {ini }}}{4 \sqrt{3 \gamma}}$,

where $\bar{t}_{\text {fin }}$ is the time of the end of inflation and $\bar{t}_{\text {ini }}$ is the time of the beginning of inflation. In the last phase

$N=\frac{1}{4 \sqrt{3}} \sqrt{\frac{1-\sqrt{1-32 \gamma \Lambda}}{\gamma}}\left(\bar{t}_{\mathrm{fin}}-\bar{t}_{\mathrm{ini}}\right)$.

Figures 9 and 10 present the number of e-folds in the first phase with respect to the parameters $\gamma$ and $\delta$. In our model, inflation appears only when $\delta \geq 0$. 


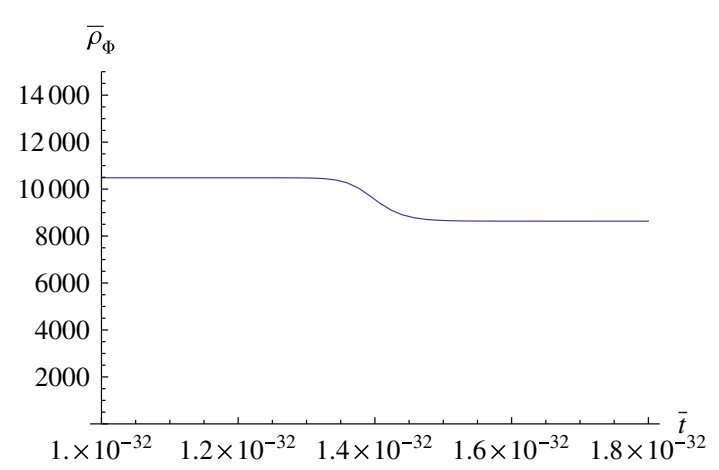

Fig. 8 The diagram presents the evolution of $\bar{\rho}_{\Phi}$ with respect to the cosmological time $\bar{t}$. The time is expressed in seconds and $\bar{\rho}_{\Phi}$ is expressed in $\frac{\mathrm{km}^{2}}{\mathrm{~s}^{2} \mathrm{Mpc}^{2}}$. The value of the parameter $\gamma$ is assumed as $3.277 \times 10^{-6} \frac{\mathrm{s}^{2} \mathrm{Mpc}^{2}}{\mathrm{~km}^{2}}$. We also assume that $\frac{\Lambda}{3 H_{0}^{2}}=0.6911$, where $H_{0}=67.74 \frac{\mathrm{km}}{\mathrm{s} \mathrm{Mpc}}$. Note that $\bar{\rho}_{\Phi}$ is not a constant function when matter is not negligible (see Fig. 4). It is interesting that the function $\bar{\rho}_{\Phi}$ is of the logistic-like function type

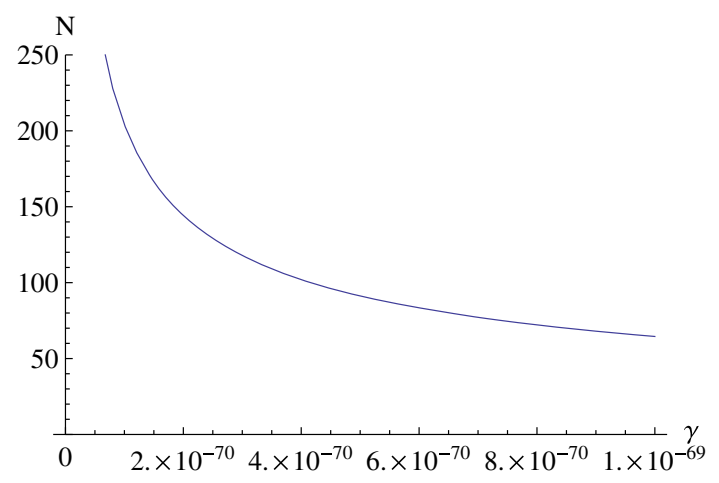

Fig. 9 The diagram presents the relation between the number of efolds $N$ and the parameter $\gamma$. The parameter $\gamma$ is given in $\frac{\mathrm{s}^{2} \mathrm{Mpc}^{2}}{\mathrm{~km}^{2}}$. We assume that $\delta=0$ and the inflation time is of order $10^{-32} \mathrm{~s}[38]$

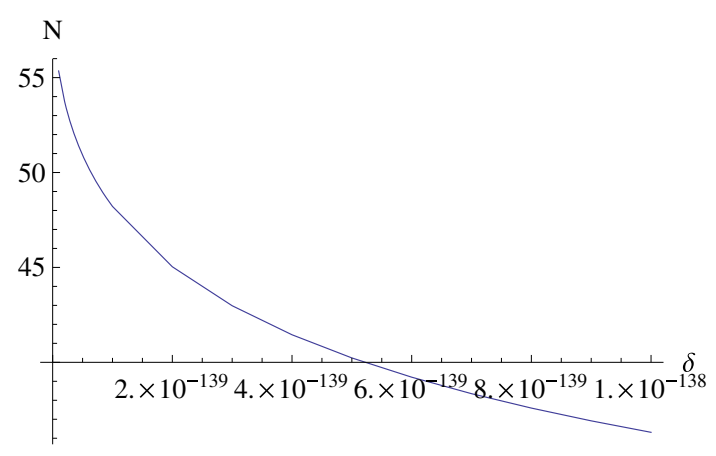

Fig. 10 The diagram presents the relation between the number of efolds $N$ and the parameter $\delta$. The parameter $\delta$ is given in $\frac{\mathrm{s}^{4} \mathrm{Mpc}^{4}}{\mathrm{~km}^{4}}$. We assume that $\gamma=1.16 \times 10^{-69} \frac{\mathrm{s}^{2} \mathrm{Mpc}^{2}}{\mathrm{~km}^{2}}$ and the inflation time is of order $10^{-32}$ s [38]

If we assume that the parameter $\delta$ is equal to zero and $N=50-60$ [37] and the inflation time is of order $10^{-32} \mathrm{~s}$
[38] then the parameter $\gamma$ belongs to the interval $(1.16 \times$ $\left.10^{-69}, 1.67 \times 10^{-69}\right)$. In consequence, the present value of $\frac{\bar{\rho}_{\phi}}{3 H_{0}^{2}}$ belongs to the interval $\left(3.41 \times 10^{-61}, 4.90 \times 10^{-61}\right)$. This means that the running dark energy is negligible in the present epoch and does not influence the acceleration of the present Universe.

If the parameter $\delta \neq 0$ the number of e-folds is modified. For the parameter $\gamma$ belonging to the interval $(1.16 \times$ $10^{-69}, 1.67 \times 10^{-69}$ ), we get the number of e-folds $N=50$ 60 , when the value of $\delta$ parameter belongs to the interval $\left(0,6.4 \times 10^{-140}\right)$.

\section{Conclusions}

We are looking for a cosmological model in which one can see both the early inflation and the late times acceleration phase of the expansion in a unique evolutional scenario. To this aim we study the cosmological model of polynomial $f(R)$ gravity cut on the $R^{3}$ term in the Palatini formalism in the Einstein frame. This model can be treated as an extension of the Starobinsky model which is formulated in the metric formalism. Our model is formulated in the Palatini formalism, but it possesses analogous features and its main advantage is simplicity. The model is reduced to the FRW model with matter and dark energy in the form of the homogeneous scalar field. Both energy densities of the matter and dark energy are determined by the Ricci scalar of the FRW metric. Therefore they are given in the covariant way. In the Einstein frame the energy density of the dark energy is fully determined by the potential of the scalar field. Because the density of dark energy is running, the interaction appears naturally between the matter and dark energy which can also be parametrized in a covariant way through the Ricci scalar. It is interesting that in our model it is possible to achieve some analytic formulae on the energy densities of dark energy and dark matter.

While the Hilbert-Einstein action and the $f(R)$-action can be related by a conformal transformation [39-41], the corresponding equations are connected by the same transformation. This fact shows that the Einstein frame and the Jordan frame are mathematically equivalent [42] but they could not be physically equivalent as pointed out in several papers (see e.g. $[41,43,44])$.

Our investigation confirms that theories equivalent mathematically on the classical level can be non-equivalent physically [45]. However, we observe in the context of our model that the Einstein frame is privileged in this sense that some strong singularities can be cured in the cosmological evolution [14]. A detailed discussion of the meaning of conformal transformations is in [46].

In our model, we have found that the plateau of the potential $\bar{U}(\Phi)$ is not necessary for the appearance of inflation 
[34]. In the expansion of the function $f(\hat{R})$, the coefficient $\delta$ of the term $\hat{R}^{3}$ affects the number of e-folds. The number of e-folds decreases for $\delta>0$ with respect to the number of e-folds obtained for the model with the $f(\hat{R})$ expansion cut off at a quadratic term. In our model, inflation appears only when $\delta \geq 0$.

In the model if the matter is vanishing we obtain eternal inflation following the stationary solution $H=$ const. This result is valid for the function $f(\hat{R})$ given by the polynomial form $f(\hat{R})=\hat{R}+\gamma \hat{R}^{2}+\sum_{i=2}^{n} \delta_{i} \hat{R}^{i+1}$. Only for an infinitesimally small fraction of matter inflation take places. The early inflation is studied in detail in terms of slow roll parameters as well as using the conception of constant roll inflation. We calculate the constant roll parameter $\beta=\frac{\mathrm{d} \ln \dot{\phi}}{\mathrm{d} \ln a}$, which measures the elasticity of $\dot{\Phi}$ with respect to the scale factor. We have found the characteristic type of the behaviour of the parameter $\beta$ following the logistic-like curve. One can distinguish four different phases in the time behaviour of the parameter $\beta$. In the first phase, the effects of matter are negligible but due to the interaction with the dark energy sector, the energy density of matter grows. As inflation progresses, matter is created, it disturbs the inflation phenomenon at the point when matter cannot be neglected. In consequence the first phase of inflation becomes unstable and the second phase appears. During the second and third phase, the effects of matter are not negligible. Finally, the fourth phase is characterized by diminishing effects of matter and the constant value of the Ricci scalar (and in consequence the constant value of energy density). During this phase dark energy dominates and the Universe behaves following the standard cosmological $\Lambda \mathrm{CDM}$ model.

Because the slow roll parameters are inadequate to constrain the model parameter we have found a bound on the model parameter $\gamma$ from the numbers of required $N$-folds. If we assume that $N=50-60$ [37] then the parameter $\gamma$ belongs to the interval $\left(1.16 \times 10^{-69}, 1.67 \times 10^{-69}\right)$. For this interval of the parameter $\gamma$, we get the number of e-folds $N=50-60$, when the value of the $\delta$ parameter belongs to the interval $\left(0,6.4 \times 10^{-140}\right)$.

Acknowledgements We are very grateful of A. Borowiec and A. Krawiec for stimulating discussion and remarks.

Open Access This article is distributed under the terms of the Creative Commons Attribution 4.0 International License (http://creativecomm ons.org/licenses/by/4.0/), which permits unrestricted use, distribution, and reproduction in any medium, provided you give appropriate credit to the original author(s) and the source, provide a link to the Creative Commons license, and indicate if changes were made. Funded by SCOAP ${ }^{3}$.

\section{References}

1. Planck Collaboration, P.A.R. Ade et al., Astron. Astrophys. 594, A13 (2016). arXiv: 1502.01589

2. A.A. Starobinsky, Phys. Lett. B 91, 99-102 (1980)

3. V.F. Mukhanov, G.V. Chibisov, JETP Lett. 33, 532-535 (1981)

4. A.A. Starobinsky, Sov. Astron. Lett. 9, 302 (1983)

5. F. Farakos, A. Kehagias, A. Riotto, Nucl. Phys. B 876, 187-200 (2013). arXiv:1307.1137

6. S. Ferrara, R. Kallosh, A. Linde, M. Porrati, JCAP 1311, 046 (2013). arXiv:1309.1085

7. Q.-G. Huang, JCAP 1402, 035 (2014). arXiv:1309.3514

8. A. Stachowski, M. Szydlowski, A. Borowiec, Eur. Phys. J. C 77, 406 (2017). arXiv: 1608.03196

9. M. Szydlowski, A. Stachowski, A. Borowiec, Eur. Phys. J. C 77, 603 (2017). arXiv: 1707.01948

10. S. Capozziello, M. De Laurentis, O. Luongo, Int. J. Mod. Phys. D 24, 1541002 (2014). arXiv:1411.2822

11. T.P. Sotiriou, V. Faraoni, Rev. Mod. Phys. 82, 451-497 (2010). arXiv:0805.1726

12. V. Faraoni, Phys. Rev. D 74, 104017 (2006). arXiv:astro-ph/0610734

13. S. Ahmad, R. Myrzakulov, M. Sami, Phys. Rev. D 96, 063515 (2017). arXiv:1705.02133

14. S.A. Appleby, R.A. Battye, A.A. Starobinsky, JCAP 1006, 005 (2010). arXiv:0909.1737

15. A. Kehagias, A.M. Dizgah, A. Riotto, Phys. Rev. D 89, 043527 (2014). arXiv:1312.1155

16. Planck Collaboration, P.A.R. Ade et al., Astron. Astrophys. 571 A22, (2014). arXiv: 1303.5082

17. Planck Collaboration, P.A.R. Ade et al., Astron. Astrophys. 594, A20 (2016). arXiv: 1502.02114

18. BICEPS2, Planck Collaboration, P.A.R. Ade et al., Phys. Rev. Lett. 114, 101301 (2015). arXiv:1502.00612

19. Planck Collaboration, R. Adam et al., Astron. Astrophys. 594, A1 (2016). arXiv: 1502.01582

20. S. Cecotti, Phys. Lett. B 190, 86-92 (1987)

21. S. Cecotti, S. Ferrara, M. Porrati, S. Sabharwal, Nucl. Phys. B 306, 160-180 (1988)

22. C. Kounnas, D. Lüst, N. Toumbas, Fortsch. Phys 63, 12-35 (2015). arXiv: 1409.7076

23. R. Blumenhagen, A. Font, M. Fuchs, D. Herschmann, E. Plauschinn, Phys. Lett. B 746, 217-222 (2015). arXiv: 1503.01607

24. J. Ellis, M.A.G. Garcia, D.V. Nanopoulos, K.A. Olive, JCAP 1510, 003 (2015). arXiv: 1503.08867

25. L. Alvarez-Gaume, A. Kehagias, C. Kounnas, D. Lüst, A. Riotto, Fortschr. Phys. 64, 176-189 (2016). arXiv:1505.07657

26. B.J. Broy, D. Ciupke, F.G. Pedro, A. Westphal, JCAP 1601, 001 (2016). arXiv:1509.00024

27. G. Allemandi, A. Borowiec, M. Francaviglia, Phys. Rev. D 70, 103503 (2004). arXiv:hep-th/0407090

28. G.J. Olmo, Int. J. Mod. Phys. D 20, 413-462 (2011). arXiv: 1101.3864

29. A. De Felice, S. Tsujikawa, Living Rev. Rel. 13, 3 (2010). arXiv: 1002.4928

30. S. Capozziello, M.F. De Laurentis, L. Fatibene, M. Ferraris, S. Garruto, SIGMA 12, 006 (2016). arXiv: 1509.08008

31. M.P. Dabrowski, J. Garecki, D.B. Blaschke, Ann. Phys. 18, 13-32 (2009). arXiv:0806.2683 
32. H. Motohashi, A.A. Starobinsky, Europhys. Lett. 117, 39001 (2017). arXiv: 1702.05847

33. H. Motohashi, A.A. Starobinsky, Eur. Phys. J. C 77, 538 (2017). arXiv: 1704.08188

34. A. Ijjas, P.J. Steinhardt, A. Loeb, Phys. Lett. B 723, 261-266 (2013). arXiv:1304.2785

35. V. Pettorino, L. Amendola, C. Wetterich, Phys. Rev. D 87, 083009 (2013). arXiv:1301.5279

36. M. Doran, G. Robbers, JCAP 0606, 026 (2006). arXiv:astro-ph/0601544

37. C. Cheng, Q.-G. Huang, Y.-Z. Ma, JCAP 1307, 018 (2013). arXiv: 1303.4497

38. BICEP2 Collaboration, P.A.R. Ade et al., Phys. Rev. Lett. 112, 241101 (2014). arXiv: 1403.3985

39. G. Allemandi, M. Capone, S. Capozziello, M. Francaviglia, Gen. Relativ. Gravity 38, 33 (2006). arXiv:hep-th/0409198
40. S. Capozziello, S. Nojiri, S.D. Odintsov, Phys. Lett. B 634, 93 (2006). arXiv:hep-th/0512118

41. S. Capozziello, S. Nojiri, S.D. Odintsov, A. Troisi, Phys. Lett. B 639, 135 (2006). arXiv:astro-ph/0604431

42. G. Magnano, L. Sokolowski, Phys. Rev. D 50, 5039 (1994). arXiv:gr-qc/9312008

43. S. Capozziello, M.F. De Laurentis, M. Francaviglia, S. Mercadante, Found. Phys. 39, 1161 (2009). arXiv:0805.3642

44. T. Clifton, Phys. Rev. D 77, 024041 (2008). arXiv:0801.0983

45. S. Capozziello, P. Martin-Moruno, C. Rubano, Phys. Lett. B 689, 117 (2010). arXiv: 1003.5394

46. S. Capozziello, M. De Laurentis, Phys. Rept. 509, 167 (2011). arXiv:1108.6266 